\title{
Macrolide antibiotics and the risk of ventricular arrhythmia in older adults
}

\author{
Mai H. Trac MSc, Eric McArthur MSc, Racquel Jandoc MSc, Stephanie N. Dixon PhD, Danielle M. Nash MSc, \\ Daniel G. Hackam MD PhD, Amit X. Garg MD PhD
}

Competing interests: Amit Garg's institution received unrestricted research funding from Pfizer. No other competing interests were declared.

This article has been peer reviewed.

Accepted: Nov. 11, 2015

Online: Feb. 22, 2016

Correspondence to: Amit Garg, amit.garg@lhsc.on.ca

CMAJ 2016. DOI:10.1503/ cmaj.150901

\begin{abstract}
- Abstract
Background: Many respiratory tract infections are treated with macrolide antibiotics. Regulatory agencies warn that these antibiotics increase the risk of ventricular arrhythmia. We examined the 30-day risk of ventricular arrhythmia and all-cause mortality associated with macrolide antibiotics relative to nonmacrolide antibiotics.
\end{abstract}

Methods: We conducted a population-based retrospective cohort study involving older adults (age > $65 \mathrm{yr}$ ) with a new prescription for an oral macrolide antibiotic (azithromycin, clarithromycin or erythromycin) in Ontario from 2002 to 2013. Our primary outcome was a hospital encounter with ventricular arrhythmia within 30 days after a new prescription. Our secondary outcome was 30-day all-cause mortality. We matched patients $1: 1$ using propensity scores to patients prescribed nonmacrolide antibiotics (amoxicillin, cefuroxime or levofloxacin). We used conditional logistic regression to measure

$\mathrm{M}$ acrolide antibiotics (e.g., azithromycin, clarithromycin and erythromycin) are a common class of medication used to treat community-acquired respiratory tract infections. $^{1-3}$ In 2010, more than 57 million outpatient prescriptions were written for macrolide antibiotics in the United States. ${ }^{4}$ An observational study prompted the US Food and Drug Administration (FDA) to issue warnings about the risk of QT interval prolongation and fatal ventricular arrhythmia with azithromycin. ${ }^{5}$ This study showed a higher risk of cardiovascular death and all-cause mortality among patients prescribed azithromycin than among patients prescribed amoxicillin or no antibiotic. ${ }^{6}$ Findings from this study were supported by case reports and other published studies. ${ }^{7-12}$ Other frequently used macrolide antibiotics, such as clarithromycin and erythromycin, have also been associated with QT interval prolongation. ${ }^{11,13,14}$

Recent studies suggest that these regulatory warnings are overstated. ${ }^{15-18}$ Mortensen and colleagues $^{15}$ showed the risk of 90-day mortality was no higher (and rather was lower) among older the association between macrolide exposure and outcomes, and repeated the analysis in 4 subgroups defined by the presence or absence of chronic kidney disease, congestive heart failure, coronary artery disease and concurrent use of a drug known to prolong the QT interval.

Results: Compared with nonmacrolide antibiotics, macrolide antibiotics were not associated with a higher risk of ventricular arrhythmia $(0.03 \%$ v. $0.03 \%$; relative risk [RR] $1.06,95 \%$ confidence interval $[\mathrm{Cl}] 0.83-1.36)$ and were associated with a lower risk of all-cause mortality $(0.62 \%$ v. $0.76 \%$; RR $0.82,95 \% \mathrm{Cl} 0.78-0.86)$. These associations were similar in all subgroups.

Interpretation: Among older adults, macrolide antibiotics were not associated with a higher 30-day risk of ventricular arrhythmia than nonmacrolide antibiotics. These findings suggest that current warnings from the US Food and Drug Administration may be overstated.

patients who received azithromycin than among those who received other guideline-concordant antibiotics. In the same study, there was no difference between the 2 groups in the risk of arrhythmia, heart failure or any cardiac event. ${ }^{15}$ Another study showed no higher 5-day risk of cardiovascular death with azithromycin than with penicillin V. ${ }^{16} \mathrm{In}$ a study involving patients with radiologically confirmed community-acquired pneumonia, compared with nonuse, clarithromycin was associated with a higher risk of cardiovascular events, but not cardiovascular mortality or all-cause mortality. ${ }^{17}$

Given the conflicting findings of prior studies, we conducted a large propensity-matched population-based cohort study involving older adults in the outpatient setting to investigate whether azithromycin, clarithromycin or erythromycin was associated with a higher 30-day risk of ventricular arrhythmia than nonmacrolide antibiotics (amoxicillin, cefuroxime or levofloxacin). Based on prior literature, we hypothesized that macrolide antibiotic use would be associated with a higher risk of ventricular arrhythmia than nonmacrolide antibiotics. 


\section{Methods}

\section{Design and setting}

We conducted a population-based retrospective cohort study involving older adults, using linked health care databases in the province of Ontario, from Apr. 1, 2002, to Mar. 1, 2013. Ontario has about 13.7 million residents, $16 \%$ of whom are aged 65 years and older. ${ }^{19}$ The Ontario Health Insurance Plan (OHIP) is the single payer for all Ontario citizens and provides universal access to hospital care and physician services. Residents aged 65 and older (about 2 million residents) also receive prescription drug coverage.

We conducted this study at the Institute for Clinical Evaluative Sciences (ICES) according to a prespecified protocol that was approved by the Research Ethics Board at Sunnybrook Health Sciences Centre (Toronto, Ontario). Participant informed consent was not required for this study. The reporting of this study followed guidelines for observational studies (Appendix 1, available at www.cmaj.ca/lookup/suppl/doi:10.1503/ cmaj.150901/-/DC1). ${ }^{20}$

\section{Data sources}

We ascertained patient characteristics, drug use, covariate information and outcome data using records from 8 databases. Vital statistics, including date of death, were obtained from the Registered Persons Database, which contains demographic information on all Ontario residents who have ever been issued a health card. We used the Ontario Drug Benefit Program database to identify prescription drug use. This database contains highly accurate records of all outpatient prescriptions dispensed to patients aged 65 and older, with an error rate of less than $1 \% .^{21} \mathrm{We}$ identified diagnostic and procedural information on all hospital admissions and emergency department visits from the Canadian Institute for Health Information (CIHI) Discharge Abstract Database and CIHI National Ambulatory Care Reporting System database. Covariate information was obtained from the OHIP database, which includes health claims for inpatient and outpatient physician services. The Canadian Organ Replacement Register identifies patients with end-stage kidney disease. Diagnostic information on all admissions to adult mental health beds was determined using the Ontario Mental Health Reporting System. The ICES Physician Database reports prescriber and specialist referral data. These data sets were linked with the use of unique, encoded identifiers and analyzed at ICES. Previous studies have used these databases to study adverse drug events and health outcomes. ${ }^{22-30}$
With the exception of prescriber specialty (missing in 19\% of prescriptions), the databases were complete for all variables used in this study.

Codes from the International Classification of Diseases, 9th revision (ICD-9; pre-2002) and the International Statistical Classification of Diseases and Related Health Problems, 10th revision (ICD-10; post-2002) were used to assess baseline comorbidities in the 5 years before receipt of the new antibiotic prescription (Appendix 2, available at www.cmaj.ca/lookup/suppl/doi:10.1503/ cmaj.150901/-/DC1). Concurrent medication use was assessed in the 120 days before cohort entry. Chest radiography, urinalysis and sputum tests were assessed in the 7 days before cohort entry. We evaluated prior health care use with physician visits, and diagnostic and screening tests performed in the previous year. Codes used to ascertain outcomes are detailed in Appendix 3, available at www.cmaj.ca/lookup/suppl/doi:10.1503/ cmaj.150901/-/DC1, which lists only ICD-10 codes because all events occurred after the implementation of this coding system.

\section{Patients}

We established a cohort of older adults in Ontario who were dispensed a new outpatient prescription for a macrolide antibiotic (azithromycin, clarithromycin or erythromycin) between Apr. 1, 2002, and Mar. 1, 2013. The date of the prescription served as the index date (referred to as the cohort entry date or start time for follow-up). These patients were compared with a group of older adults with similar baseline indicators of health who were prescribed nonmacrolide antibiotics that have similar indications for use as macrolide antibiotics but have not been found to be associated with ventricular arrhythmia (amoxicillin or cefuroxime) or have weak proarrhythmic potential (levofloxacin). ${ }^{14}$

Before matching, we excluded the following patients from both groups: those who were in their first year of eligibility for prescription drug coverage (age $65 \mathrm{yr}$ ), to avoid incomplete medication records; those with prescriptions for any antibiotic in the 180 days before the index date, to ensure that the drug was newly prescribed; those who received a prescription for more than 1 type of antibiotic on the index date, to form mutually exclusive groups; those who were discharged from the hospital in the 2 days before their index date, to ensure these were new outpatient antibiotic prescriptions (because in Ontario, patients continuing an antibiotic treatment initiated in the hospital would have their outpatient antibiotic prescription dispensed on the same day or the day after hospital discharge); and those with daily drug doses that were not standard for the treatment of respiratory tract infections, to ensure generalizability to usual prescribing (Appendix 4, 
available at www.cmaj.ca/lookup/suppl/doi: 10.1503/cmaj.150901/-/DC1). Study patients could enter the cohort only once, so for patients with multiple eligible prescriptions, we restricted the information to the first eligible prescription.

\section{Outcomes}

The primary outcome of this study was a hospital encounter (presentation to the emergency department or hospital admission) with ventricular arrhythmia (ventricular tachycardia or ventricular fibrillation). The secondary outcome was all-cause mortality. We restricted our analysis to 30 days after the index date because macrolide antibiotics are prescribed for short durations and any observed outcomes can be reasonably attributed to the study drugs over this time frame. Furthermore, QT prolongation has been shown to start within hours to days of initiating macrolides, and thus, one would expect that drug-related ventricular arrhythmias would occur soon after initiating a macrolide antibiotic prescription. ${ }^{11,12,31-33}$ We conducted a secondary analysis using a 14-day (v. 30-d) follow-up.

The diagnosis codes used to identify ventricular arrhythmia are presented in Appendix 3. Trained personnel enter these codes into the databases based only on physician-recorded diagnoses in a patient's medical chart. The ICD-10 codes for ventricular arrhythmia have not been fully validated; however, their sensitivity would be expected to be low, since true ventricular arrhythmias frequently go undetected in routine health care (because they often occur outside hospital settings, in unmonitored patients in hospital or in a setting of multiorgan medical illness). In previous studies assessing the accuracy of ICD-9 and ICD-10 codes for cardiac arrhythmia (ventricular and supraventricular), the positive predictive value (truly have the condition when code is present) exceeded $80 \% .^{34-37}$ We performed a manual review of 202 charts in our region and confirmed a positive predictive value of $92 \%$ (95\% confidence interval [CI] 87\%95\%) for the set of codes used in this study to detect ventricular arrhythmia. Vital statistics for allcause mortality are coded accurately in our data sources, with a sensitivity of $97.8 \%$ and specificity of $100 \%$ for the finding of death..$^{38}$ Because codes to assess specific cause of death in our region have not been validated and are expected to be inaccurate, we did not include this outcome in our study.

\section{Statistical analysis}

We used propensity score matching to eliminate systematic differences in the measured baseline characteristics of our comparison groups. This allowed us to form a matched set of patients in our 2 groups with a similar probability of receiving a macrolide antibiotic given a set of measured base- line covariates. ${ }^{39,40} \mathrm{We}$ estimated the propensity scores using a multivariable logistic regression model with 106 baseline characteristics selected because of their potential influence on the outcomes or segregation of patients between the comparison groups (Appendix 5, available at www. cmaj.ca/lookup/suppl/doi:10.1503/cmaj.150901/-/ DC1). ${ }^{40-42}$ We matched patients in the macrolide antibiotic group with patients in the nonmacrolide antibiotic group in a 1:1 ratio using greedy matching with a caliper width within 0.2 standard deviations of the logit of the propensity score. We also matched patients based on presence or absence of the following 4 conditions to conduct prespecified subgroup analyses for the matched pairs: chronic kidney disease, congestive heart failure, coronary artery disease and concurrent use of a drug known to prolong the QT interval (Appendix 6, available at www.cmaj.ca/lookup/suppl/doi:10.1503/ cmaj.150901/-/DC1). We compared baseline characteristics between those prescribed macrolide antibiotics and those prescribed nonmacrolide antibiotics using standardized differences. This metric describes differences between group means relative to the pooled standard deviation and is considered a meaningful difference if greater than $10 \%{ }^{43}$ We estimated the odds ratio (OR) and 95\% CIs for the primary and secondary outcomes using conditional logistic regression, which accounted for matching. ${ }^{44}$ Each OR was approximated to be the relative risk (RR) (appropriate given the incidences observed). We also performed multivariable logistic regression with the unmatched cohort, adjusting for age, sex and the 4 subgroup conditions defined above.

We evaluated the association between macrolide antibiotic use and our outcomes in 4 specified subgroups within our propensitymatched cohort, as defined above. We hypothesized that any RR of ventricular arrhythmia associated with macrolide antibiotics compared with nonmacrolide antibiotics might be greater when these conditions were present than when they were absent. For example, the dose of clarithromycin should be reduced by $50 \%$ in chronic kidney disease owing to impaired clearance, but in practice this seldom occurs. ${ }^{45}$ Similarly, in prior reports, ventricular arrhythmia associated with macrolide antibiotic use occurred in patients with pre-existing risk factors, such as congestive heart failure ${ }^{46}$ and cardiovascular disease, ${ }^{11,47-49}$ and those taking other QTprolonging medications. ${ }^{50,51} \mathrm{We}$ also assessed interaction by type of macrolide antibiotic, in which patients taking nonmacrolide antibiotics followed their matched pair. We determined interaction $p$ values by including interaction terms in the regression models. In addition, 
because levofloxacin has been associated with weak proarrhythmic potential, we repeated our analyses excluding matched pairs that included levofloxacin, to determine whether this affected the risk for ventricular arrhythmia associated with macrolide antibiotics compared with nonmacrolide antibiotics. We conducted all analyses with SAS version 9.4. In all outcome analyses, we interpreted 2-tailed $p$ values less than 0.05 as significant.

\section{Role of the funding source}

The study design and conduct, opinions, results and conclusions in this article are those of the authors and are independent of the funding sources.

\section{Results}

\section{Baseline characteristics}

Cohort selection is presented in Appendix 7 (available at www.cmaj.ca/lookup/suppl/doi

Table 1 (part 1 of 2): Selected baseline characteristics of patients taking macrolide and nonmacrolide antibiotics before and after matching

\begin{tabular}{|c|c|c|c|c|c|c|}
\hline \multirow[b]{2}{*}{ Characteristic } & \multicolumn{3}{|c|}{ Unmatched, no. (\%) of patients* } & \multicolumn{3}{|c|}{ Matched, no. (\%) of patients* } \\
\hline & $\begin{array}{c}\text { Macrolide } \\
n=616359\end{array}$ & $\begin{array}{c}\text { Nonmacrolide } \\
n=705132\end{array}$ & $\begin{array}{l}\text { Standardized } \\
\text { difference, } \uparrow \%\end{array}$ & $\begin{array}{c}\text { Macrolide } \\
n=503612\end{array}$ & $\begin{array}{c}\text { Nonmacrolide } \\
n=503612\end{array}$ & $\begin{array}{r}\text { Standardized } \\
\text { difference, } † \%\end{array}$ \\
\hline \multicolumn{7}{|l|}{ Demographics } \\
\hline Age, mean $\pm S D, y r$ & $73.7 \pm 7.1$ & $74.1 \pm 7.3$ & 6 & $73.9 \pm 7.2$ & $74.0 \pm 7.2$ & 2 \\
\hline Sex, female & $359895(58.4)$ & $389520(55.2)$ & 6 & $288515(57.3)$ & $288473(57.3)$ & 0 \\
\hline \multicolumn{7}{|l|}{ Income quintilef } \\
\hline 1 (low) & $120433(19.5)$ & $135873(19.3)$ & 1 & 99340 (19.7) & $100535(20.0)$ & 1 \\
\hline 2 & $128791(20.9)$ & $147680(20.9)$ & 0 & $106101(21.1)$ & $106738(21.2)$ & 0 \\
\hline 3 (middle) & $121353(19.7)$ & $139156(19.7)$ & 0 & $101012(20.1)$ & $101455(20.1)$ & 0 \\
\hline 4 & $120224(19.5)$ & $138126(19.6)$ & 0 & 97895 (19.4) & $97247(19.3)$ & 0 \\
\hline 5 (high) & $123398(20.0)$ & $141829(20.1)$ & 0 & 99264 (19.7) & $97637(19.4)$ & 1 \\
\hline Rural residence§ & $87490(14.2)$ & $89820(12.7)$ & 4 & 68952 (13.7) & 67861 (13.5) & 1 \\
\hline Long-term care & 20921 & $37020 \quad$ (5.3) & 9 & $20538 \quad(4.1)$ & 23129 (4.6) & 3 \\
\hline \multicolumn{7}{|l|}{ Prescribing physicianๆ } \\
\hline General practitioner & $474660(77.0)$ & 398920 (56.6) & 44 & 367302 (72.9) & 368847 (73.2) & 1 \\
\hline Internist & $2797 \quad(0.5)$ & $2967 \quad(0.4)$ & 1 & $2461 \quad(0.5)$ & $2611 \quad(0.5)$ & 0 \\
\hline Nephrologist & $802 \quad(0.1)$ & $788 \quad(0.1)$ & 1 & $749 \quad(0.1)$ & $626 \quad(0.1)$ & 1 \\
\hline Cardiologist & $856 \quad(0.1)$ & $1146 \quad(0.2)$ & 1 & $813 \quad(0.2)$ & $909 \quad(0.2)$ & 0 \\
\hline Other & 37133 (6.0) & $38396 \quad(5.4)$ & 2 & 32194 (6.4) & $34646 \quad(6.9)$ & 2 \\
\hline \multicolumn{7}{|l|}{ Comorbidities** } \\
\hline $\begin{array}{l}\text { Chronic kidney } \\
\text { diseaset† }\end{array}$ & $27800 \quad(4.5)$ & $36539 \quad(5.2)$ & 3 & $25543 \quad(5.1)$ & $25543 \quad(5.1)$ & 0 \\
\hline $\begin{array}{l}\text { Congestive heart } \\
\text { failure }\end{array}$ & $61351(10.0)$ & $77802(11.0)$ & 4 & $56214(11.2)$ & $56214(11.2)$ & 0 \\
\hline $\begin{array}{l}\text { Coronary artery } \\
\text { disease } \neq \neq\end{array}$ & $158521(25.7)$ & $190688(27.0)$ & 3 & $138038(27.4)$ & $138038(27.4)$ & 0 \\
\hline Angina & $117261(19.0)$ & $135415(19.2)$ & 0 & 99400 (19.7) & 99463 (19.7) & 0 \\
\hline Chronic lung disease & $177653(28.8)$ & $167962(23.8)$ & 11 & $138346(27.5)$ & $136245(27.1)$ & 1 \\
\hline $\begin{array}{l}\text { Atrial fibrillation or } \\
\text { flutter }\end{array}$ & 25513 (4.1) & $39678 \quad(5.6)$ & 7 & $23970 \quad(4.8)$ & $25574 \quad(5.1)$ & 1 \\
\hline Cancer§§ & $76143(12.4)$ & $86596(12.3)$ & 0 & 62477 (12.4) & $62302(12.4)$ & 0 \\
\hline \multicolumn{7}{|c|}{ Charlson Comorbidity Indexףा } \\
\hline 0 & $394182(64.0)$ & $441541(62.6)$ & 3 & $396258(78.7)$ & $394517(78.3)$ & 1 \\
\hline 1 & $151182(24.5)$ & $173421(24.6)$ & 0 & 44718 (8.9) & $45227 \quad(9.0)$ & 0 \\
\hline 2 & $36695 \quad(6.0)$ & $44093 \quad(6.3)$ & 1 & $31456 \quad(6.2)$ & 31689 (6.3) & 0 \\
\hline$\geq 3$ & $34300 \quad(5.6)$ & $46077 \quad(6.5)$ & 4 & $31180 \quad(6.2)$ & 32179 (6.4) & 1 \\
\hline
\end{tabular}


:10.1503/cmaj.150901/-/DC1), and selected baseline characteristics before and after matching are presented in Table 1 (for the full list, see Appendix 8, available at www.cmaj.ca/lookup/suppl/ doi:10.1503/cmaj.150901/-/DC1). We identified 616359 older adults with prescriptions for macrolide antibiotics $(n=296121$ for azithromycin, $n=301127$ for clarithromycin and $n=19111$ for erythromycin) and 705132 patients with prescriptions for nonmacrolide antibiotics $(n=$ 556790 for amoxicillin, $n=72701$ for cefuroxime and $n=75641$ for levofloxacin) before matching. The mean age of the entire cohort was 74 years, and $57 \%$ were women.

Table 1 (part 2 of 2): Selected baseline characteristics of patients taking macrolide and nonmacrolide antibiotics before and after matching

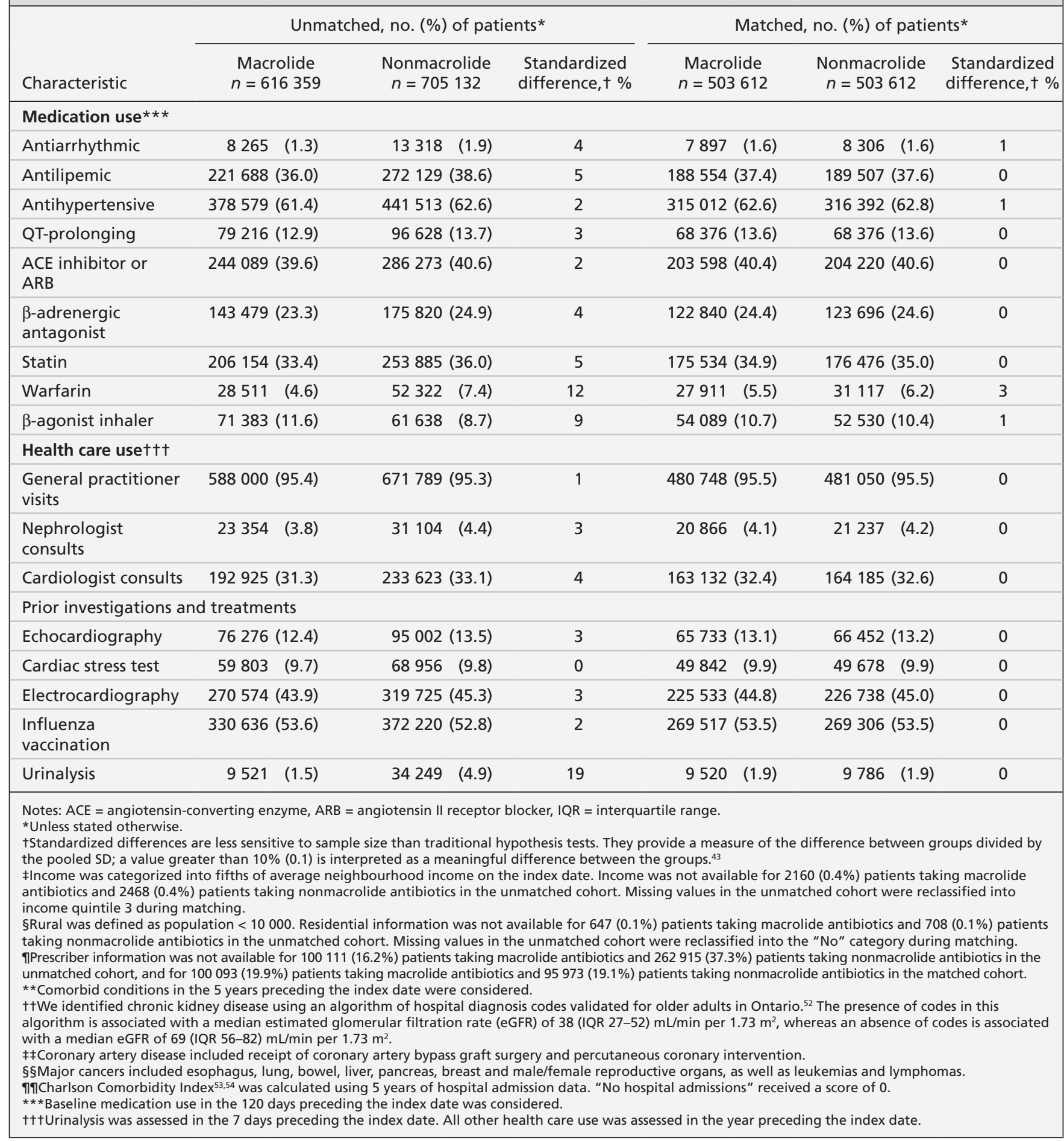


Before matching, patients prescribed macrolide antibiotics were more likely to receive their prescription from a general practitioner $\mathbf{7 7 . 0 \%}$ v. $56.6 \%)$ and have chronic lung disease $(28.8 \%$ v. $23.8 \%$ ) than patients prescribed nonmacrolide antibiotics, and were less likely to take warfarin $(4.6 \%$ v. $7.4 \%)$ and have a urinalysis test $(1.5 \%$ v. $4.9 \%$ ). A total of 503612 patients taking macrolide antibiotics were successfully matched with 503612 patients taking nonmacrolide antibiotics. The 2 groups were well-balanced and showed no meaningful differences in the 106 measured baseline characteristics (Appendix 8). Clarithromycin and azithromycin were the most frequently prescribed macrolide antibiotics ( $48.9 \%$ and $48.0 \%$, respectively), followed by erythromycin $(3.1 \%)$. The median starting daily dose for clarithromycin was 1000 (interquartile range [IQR] 500-1000) $\mathrm{mg}$; for azithromycin, 300 (IQR 300-300) mg; and for erythromycin, 1000 (IQR 999-1000) mg. The median day supply for clarithromycin was 10 (IQR 7-10) days; for azithromycin, 5 (IQR 5-5) days; and for erythromycin, 7 (IQR 7-10) days. General practitioners were the most frequent prescribers (73\% in both groups). The region of Ontario where the antibiotic was prescribed was also well-balanced among matched patients taking macrolide and nonmacrolide antibiotics. The interaction by macrolide type was not significant $(p=0.7)$, thus macrolides were examined as a group in all analyses.

Table 2: Event rates for 30-day hospital encounter with ventricular arrhythmia and all-cause mortality in a matched cohort of patients prescribed macrolide and nonmacrolide antibiotics

\begin{tabular}{|c|c|c|c|c|}
\hline \multirow[b]{2}{*}{ Outcome } & \multicolumn{2}{|c|}{ No. $(\%)$ of patients } & \multirow[b]{2}{*}{ Relative risk $(95 \% \mathrm{CI})$} & \multirow[b]{2}{*}{$p$ value } \\
\hline & $\begin{array}{c}\text { Macrolide } \\
n=503612\end{array}$ & $\begin{array}{c}\text { Nonmacrolide } \\
n=503612\end{array}$ & & \\
\hline Ventricular arrhythmia* & $134(0.03)$ & $126(0.03)$ & $1.06(0.83-1.36)$ & 0.6 \\
\hline All-cause mortality & $3144(0.62)$ & $3833(0.76)$ & $0.82(0.78-0.86)$ & $<0.001$ \\
\hline \multicolumn{5}{|c|}{$\begin{array}{l}\text { Note: } \mathrm{Cl}=\text { confidence interval. } \\
\text { *Based on hospital presentation (emergency department or hospital admission) assessed by hospital diagnosis codes. This } \\
\text { method of assessment underestimated the true number of events because ventricular arrhythmias frequently go undetected in } \\
\text { routine health care (because they often occur outside of hospital settings, in unmonitored patients in hospital or in a setting of } \\
\text { multiorgan medical illness in which recorded codes describe illnesses other than ventricular arrhythmia). }\end{array}$} \\
\hline
\end{tabular}

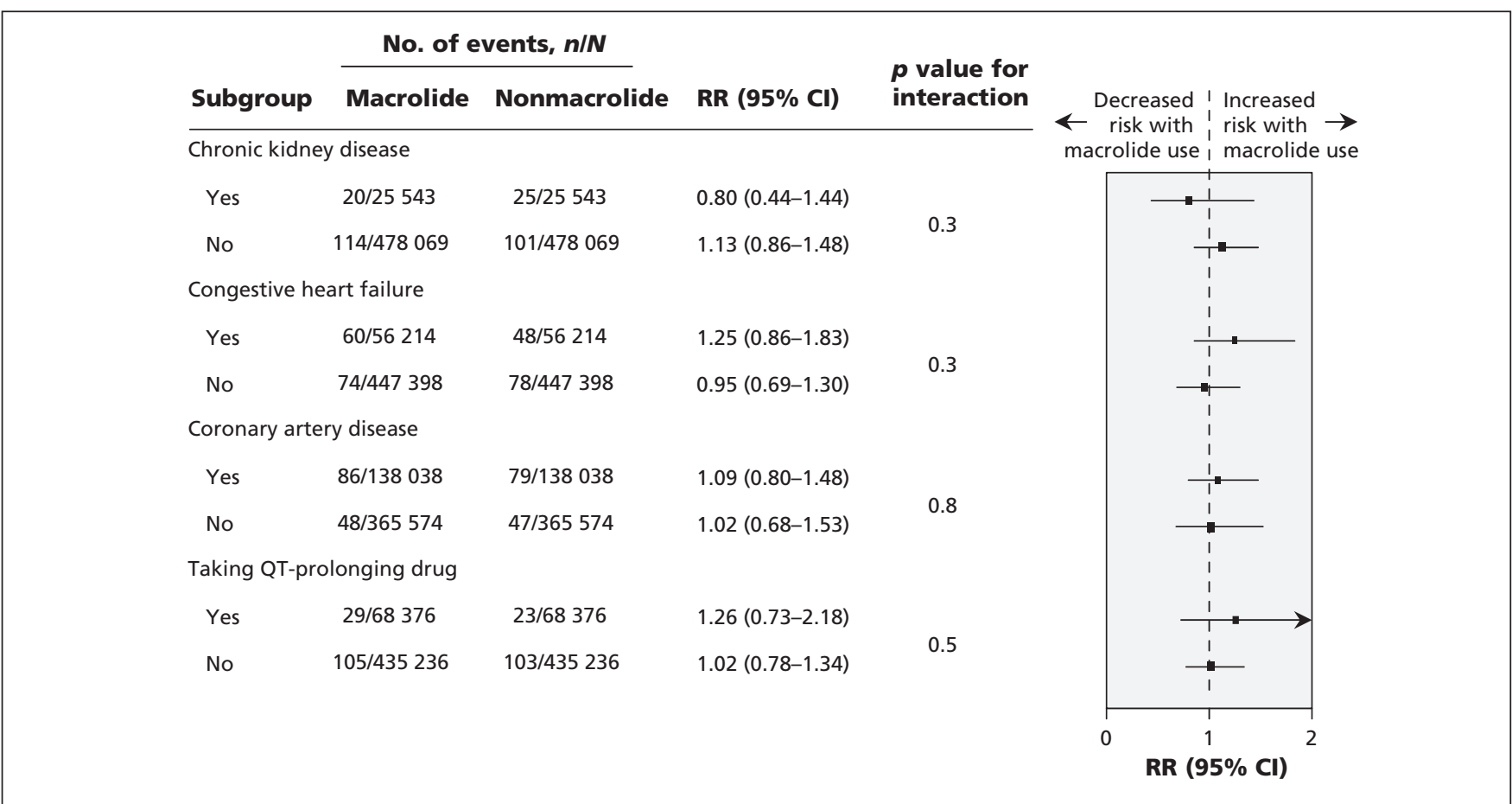

Figure 1: Risk of 30-day hospital encounter with ventricular arrhythmia* associated with use of a macrolide antibiotic (azithromycin, clarithromycin or erythromycin) examined in subgroups defined by chronic kidney disease, congestive heart failure, coronary artery disease and use of a QT-prolonging drug. Data marker size is proportional to the inverse of the source variance. *Based on hospital presentation (emergency department visit or hospital admission) assessed by hospital diagnosis codes. Note: $\mathrm{Cl}=$ confidence interval, $\mathrm{RR}=$ relative risk. 


\section{Outcomes}

The primary and secondary outcomes are shown in Table 2. Across the entire cohort, during the 30-day follow-up period, 260 patients $(0.03 \%)$ had a record of a hospital encounter with ventricular arrhythmia and 6977 (0.69\%) died.

The 30-day risk of ventricular arrhythmia associated with macrolide antibiotics compared with nonmacrolide antibiotics was not significantly different $(0.03 \%$ v. $0.03 \%$; RR $1.06,95 \%$ CI $0.83-1.36 ; p=0.6$ ). Use of macrolide antibiotics was associated with a lower risk of all-cause mortality $(0.62 \%$ v. $0.76 \%$; RR 0.82 , CI 0.78 0.86; $p<0.001)$. Similar results were observed with use of multivariable logistic regression in our entire unmatched cohort (Appendix 9, available at www.cmaj.ca/lookup/suppl/doi:10.1503/ cmaj.150901/-/DC1).

\section{Subgroup analyses}

Subgroup analyses for ventricular arrhythmia and all-cause mortality are shown in Figures 1 and 2. The presence or absence of chronic kidney disease, congestive heart failure, coronary artery disease or concurrent use of a QT-prolonging drug did not significantly modify the relative association between antibiotic exposure and the risk of ventricular arrhythmia ( $p$ values for the interaction ranged from 0.3 to 0.8 ). Across all subgroups, use of macrolide antibiotics was associated with a lower risk of all-cause mortality than use of nonmacrolide antibiotics, with no modification of the relative association across subgroups ( $p$ values for the interaction ranged from 0.1 to 0.7 ).

\section{Additional analyses}

First, we examined our outcomes at 14 days (rather than $30 \mathrm{~d}$ ) of follow-up. Similar to our 30-day follow-up analysis, macrolide antibiotic use was associated with no different risk in ventricular arrhythmia and a lower risk of allcause mortality than nonmacrolide antibiotic use (Appendix 10, available at www.cmaj.ca/ lookup/suppl/doi:10.1503/cmaj.150901/-/DC1).

Second, when we repeated our analyses excluding matched pairs taking levofloxacin, the association with ventricular arrhythmia did not change $(0.03 \%$ v. $0.02 \%$; RR $1.21,95 \%$ CI $0.92-$ $1.58 ; p=0.2)$.

\section{Interpretation}

In this population-based cohort study, we observed that, compared with nonmacrolide antibiotics, new use of macrolide antibiotics was associated with a similar 30-day risk of ventricular arrhythmia $(0.03 \%$ v. $0.03 \%)$ and a slightly lower risk of allcause mortality ( $0.62 \%$ v. $0.76 \%)$.

The findings of 9 published studies (summarized in Appendix 11, available at www.cmaj.ca/ lookup/suppl/doi:10.1503/cmaj.150901/-/DC1) describing the risk of arrhythmia or death with use of macrolide antibiotics compared with other classes of antibiotics or nonuse are inconsistent.

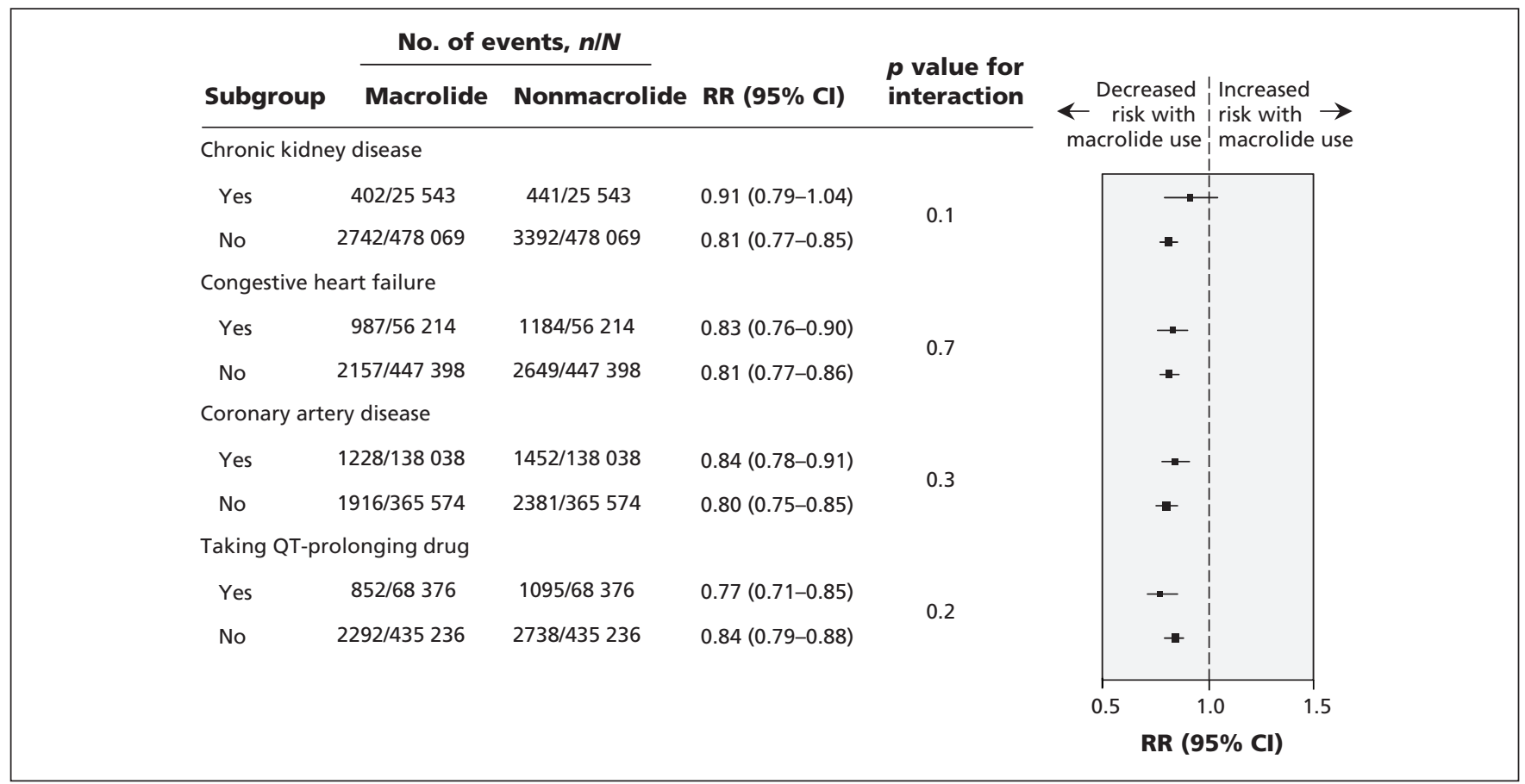

Figure 2: Risk of 30-day all-cause mortality associated with use of a macrolide antibiotic (azithromycin, clarithromycin or erythromycin) examined in subgroups defined by chronic kidney disease, congestive heart failure, coronary artery disease and use of a QT-prolonging drug. Data marker size is proportional to the inverse of the source variance. Note: $\mathrm{Cl}=$ confidence interval, $\mathrm{RR}=$ relative risk. 
These studies vary in patient age, sex and comorbidity. Two studies reported no significant increase in the risk of ventricular arrhythmia associated with macrolide use relative to other antibiotics after $10^{7}$ and $30^{15}$ days of follow-up, although Rao and colleagues ${ }^{7}$ reported an increased risk during the first 5 days of treatment only. The 7 remaining studies did not examine arrhythmia risk; however, 1 study did report a higher risk of cardiac events. ${ }^{17}$ With respect to our secondary outcome of allcause mortality, 2 studies reported a higher risk associated with macrolide antibiotic use $\mathrm{e}^{6,9}$ and 3 other studies reported no such risk. ${ }^{15,17,18}$ Similar to our results, 2 studies reported macrolide antibiotics to be associated with a lower risk of all-cause mortality than nonmacrolide antibiotics. ${ }^{15,18}$ Rao and colleagues ${ }^{7}$ reported an increased risk of allcause mortality during the first 5 days of treatment, but not for treatment days 6 to 10 . We used the quality checklist developed by Downs and Black ${ }^{55}$ to assess the reporting, external validity, internal validity and statistical power of the prior 9 studies (Appendix 12, available at www.cmaj.ca/lookup/ suppl/doi:10.1503/cmaj.150901/-/DC1). Six of the reviewed studies were of fair quality, ${ }^{6-8,15,17,56}$ and 3 were of good quality. ${ }^{9,16,18}$

Of particular interest is the comparison of our findings with those of Ray and colleagues, ${ }^{6}$ who observed a higher 5-day risk of cardiovascular death (hazard ratio [HR] 2.88, 95\% CI 1.79-4.63) and death from any cause (HR 1.85, 95\% CI 1.25-2.75) among patients taking azithromycin compared with patients not taking an antibiotic, as well as among patients taking azithromycin compared with those taking amoxicillin (HR 2.49, 95\% CI 1.38-4.50, for cardiovascular death; HR $2.02,95 \%$ CI $1.24-3.30$, for all-cause mortality). Although the authors matched baseline characteristics when they compared azithromycin use with nonuse, no matching was done when the reference group was use of amoxicillin. Compared with the azithromycin group, patients receiving amoxicillin seemed healthier overall, with a lower proportion of baseline medication use (with the exception of digoxin and insulin), a lower prevalence of comorbidities and fewer hospital admissions and emergency department visits. Accordingly, fewer deaths may have occurred among those receiving amoxicillin if they were less prone to events than the macrolide antibiotic group.

In this same study, the authors reported the greatest risk of cardiovascular death among patients with the highest baseline risk of cardiovascular disease. ${ }^{6}$ Additionally, Schembri and colleagues ${ }^{17}$ reported an increased risk of cardiovascular events with prolonged courses of clarithromycin (>7d), especially in patients with pre-existing coronary artery disease. For these reasons, we explored congestive heart failure and coronary artery disease as subgroups in our study, in addition to chronic kidney disease and use of other QT-prolonging medications. In contrast to prior studies, we found that these variables did not significantly alter the association between macrolide antibiotic use and our outcomes. Nonetheless, these findings should be interpreted with caution, and physicians should always consider a patient's baseline risk for adverse events before prescribing macrolides or other antibiotics.

Three recent reviews of case reports have identified several major risk factors for macrolideinduced QT prolongation: existing QT interval prolongation, a history of torsades de pointes, female sex, older age, heart disease, hypokalemia, hypomagnesemia, significant bradycardia, bradyarrhythmias and use of QT-prolonging drugs and metabolic inhibitors. ${ }^{57-59}$ Progression of QT prolongation to more serious cardiac events commonly occurs when many of these clinical risk factors are present together. Older women with heart disease are at particularly higher risk..$^{57}$ To minimize risk, others advocate for electrocardiography to monitor the QT interval before and after initiating therapy. ${ }^{60}$ There has also been recent speculation about the role of potential genetic modifiers in clarithromycin-related arrhythmia risk, and some believe genetic screening for potential druginduced QT prolongation may be of value. ${ }^{61}$

\section{Strengths and limitations}

Our study has several strengths. The use of Ontario's health care databases with data on universal prescription drug coverage for older adults provided us with a large representative sample of patients who received the study antibiotics in routine care. This allowed us to estimate the risks of uncommon but serious adverse events with good precision and external validity. We assessed clinically important adverse events (hospital encounter with ventricular arrhythmia and death), rather than relying on surrogate outcomes such as a prolonged QT interval on an electrocardiogram, which makes these findings of particular interest to clinicians and regulatory agencies. In addition, we were able to reduce the influence of confounding by indication, a bias common to observational drug studies. Confounding by indication assumes that patients who receive a drug for a particular reason may have an altered risk of study outcomes because of this underlying reason compared with those who did not receive the drug. ${ }^{62}$ To reduce the influence of confounding by indication, we compared patients prescribed macrolide antibiotics with patients prescribed nonmacrolide antibiotics (amoxicillin, cefuroxime or levofloxacin) that have similar indications (e.g., treatment of community-acquired 
respiratory tract infections) as macrolide antibiotics but are not meaningfully associated with a higher risk of ventricular arrhythmia.

Our study has some limitations. Prospective data collection with independent outcome adjudication would be a preferred method to assess risk. In this study, we analyzed retrospective data using administrative diagnosis codes assigned from physician records, and cardiac rhythm tracings were not available in our data sources. The diagnosis codes we used for a hospital encounter with ventricular arrhythmia have a good positive predictive value but limited sensitivity. However, we have no reason to suspect any systematic difference in diagnosis recording by antibiotic type, which suggests that our relative measures of risk are robust. In addition, we were unable to determine the specific indication for which the antibiotics were prescribed, because this information is not captured in our pharmacy database. However, we did know the specialty of the prescriber, which was similar in our 2 matched groups. As with all observational studies, residual confounding can never be eliminated; however, we used a statistical technique to ensure our comparison groups were similar on 106 measured baseline characteristics. Our findings may not be generalizable to other regions if Ontario physicians deliberately avoided prescribing macrolide antibiotics to patients at highest risk of ventricular arrhythmia (e.g., those with baseline QT prolongation) or discontinued macrolide antibiotics when the QT interval was prolonged in a follow-up electrocardiogram. However, our impression is that most Ontario physicians rarely take ventricular arrhythmia risk into account when deciding to prescribe a macrolide. Although shorter follow-up periods were used in prior studies, ${ }^{6-8,16,17,56}$ we consider the 30-day follow-up used in our study to be clinically relevant. We did, however, repeat our analyses within 14 days of macrolide prescription and found similar results.

\section{Implications for practice}

This study was prompted by FDA warnings about a higher risk of QT interval prolongation and subsequent ventricular arrhythmia among patients taking azithromycin. ${ }^{5}$ Although caution should be exercised when prescribing macrolide antibiotics to high-risk patients in whom drug clearance or electrical activity of the heart is impaired and outcomes less predictable, our study findings and examination of the current literature suggest that the risk of ventricular arrhythmia and death from macrolide antibiotic use may be overstated. A careful re-examination and updating of drug prescribing references ${ }^{45,63-65}$ and warnings from regulatory agencies may be warranted.

\section{Conclusion}

Among older adults prescribed macrolide antibiotics compared with nonmacrolide antibiotics, we found no difference in risk of a hospital encounter with ventricular arrhythmia within 30 days of a new prescription and a lower risk of 30-day all-cause mortality. These findings are reassuring for health care providers who prescribe macrolide antibiotics to a wide range of patients in routine care.

\section{References}

1. Medicines use and spending shifts: a review of the use of medicines in the US in 2014. New Jersey: IMS Institute for Healthcare Informatics; 2015

2. Prescribed drug spending in Canada, 2013: a focus on public drug programs - Top 100 drug classes, data tables. Ottawa: Canadian Institute for Health Information; 2015.

3. File TM Jr. Treatment of community-acquired pneumonia in adults in the outpatient setting. UpToDate; 2015.

4. Hicks LA, Taylor TH Jr, Hunkler R. US outpatient antibiotic prescribing, 2010. N Engl J Med 2013;368:1461-2.

5. Azithromycin (Zithromax or Zmax): drug safety communication - risk of potentially fatal heart rhythms. Maryland: US Food and Drug Administration; 2013.

6. Ray WA, Murray KT, Hall K, et al. Azithromycin and the risk of cardiovascular death. N Engl J Med 2012;366:1881-90.

7. Rao GA, Mann JR, Shoaibi A, et al. Azithromycin and levofloxacin use and increased risk of cardiac arrhythmia and death. Ann Fam Med 2014;12:121-7.

8. Ray WA, Murray KT, Meredith S, et al. Oral erythromycin and the risk of sudden death from cardiac causes. $N$ Engl J Med 2004;351:1089-96.

9. Jespersen CM, Als-Nielsen B, Damgaard M, et al. Randomised placebo controlled multicentre trial to assess short term clarithromycin for patients with stable coronary heart disease: CLARICOR trial. BMJ 2006;332:22-7.

10. Cetin M, Yildirimer M, Ozen S, et al. Clarithromycin-induced long QT syndrome: a case report. Case Rep Med 2012;2012:634652.

11. Matsunaga N, Oki Y, Prigollini A. A case of QT-interval prolongation precipitated by azithromycin. N Z Med J 2003;116:U666.

12. Huang $\mathrm{BH}, \mathrm{Wu} \mathrm{CH}$, Hsia $\mathrm{CP}$, et al. Azithromycin-induced torsade de pointes. Pacing Clin Electrophysiol 2007;30:1579-82.

13. Bril F, Gonzalez CD, Di Girolamo G. Antimicrobial agents-associated with QT interval prolongation. Curr Drug Saf 2010;5:85-92.

14. Owens RC, Nolin TD. Antimicrobial-associated QT interval prolongation: pointes of interest. Clin Infect Dis 2006;43:1603-11.

15. Mortensen EM, Halm EA, Pugh MJ, et al. Association of azithromycin with mortality and cardiovascular events among older patients hospitalized with pneumonia. JAMA 2014;311:2199-208.

16. Svanström H, Pasternak B, Hviid A. Use of azithromycin and death from cardiovascular causes. N Engl J Med 2013;368:1704-12.

17. Schembri S, Williamson PA, Short PM, et al. Cardiovascular events after clarithromycin use in lower respiratory tract infections: analysis of two prospective cohort studies. BMJ 2013;346:f1235.

18. Asadi L, Eurich DT, Gamble JM, et al. Guideline adherence and macrolides reduced mortality in outpatients with pneumonia. Respir Med 2012;106:451-8.

19. Population by sex and age group, by province and territory (Number, female) [table]. Ottawa: Statistics Canada; 2015.

20. von Elm E, Altman DG, Egger M, et al. The Strengthening the Reporting of Observational Studies in Epidemiology (STROBE) statement: guidelines for reporting observational studies. J Clin Epidemiol 2008;61:344-9.

21. Levy AR, O'Brien BJ, Sellors C, et al. Coding accuracy of administrative drug claims in the Ontario Drug Benefit database. Can J Clin Pharmacol 2003;10:67-71.

22. Patel AM, Shariff S, Bailey DG, et al. Statin toxicity from macrolide antibiotic coprescription: a population-based cohort study. Ann Intern Med 2013;158:869-76.

23. Zhao YY, Weir MA, Manno M, et al. New fibrate use and acute renal outcomes in elderly adults: a population-based study. Ann Intern Med 2012;156:560-9.

24. Shih AW, Weir MA, Clemens KK, et al. Oral bisphosphonate use in the elderly is not associated with acute kidney injury. Kidney Int 2012;82:903-8.

25. Molnar AO, Coca SG, Devereaux PJ, et al. Statin use associates with a lower incidence of acute kidney injury after major elective surgery. J Am Soc Nephrol 2011;22:939-46. 
26. Gandhi S, Fleet JL, Bailey DG, et al. Calcium-channel blockerclarithromycin drug interactions and acute kidney injury. JAMA 2013;310:2544-53.

27. Siddiqui NF, Coca SG, Devereaux PJ, et al. Secular trends in acute dialysis after elective major surgery - 1995 to 2009. CMAJ 2012;184:1237-45.

28. Jain AK, Cuerden MS, McLeod I, et al. Reporting of the estimated glomerular filtration rate was associated with increased use of angiotensin-converting enzyme inhibitors and angiotensin-II receptor blockers in CKD. Kidney Int 2012;81:1248-53.

29. Li DQ, Kim R, McArthur E, et al. Risk of adverse events among older adults following co-prescription of clarithromycin and statins not metabolized by cytochrome P450 3A4. CMAJ 2015; $187: 174-80$

30. Weir MA, Beyea MM, Gomes T, et al. Orlistat and acute kidney injury: an analysis of 953 patients. Arch Intern Med 2011 171:703-4

31. Howard PA. Azithromycin-induced proarrhythmia and cardiovascular death. Ann Pharmacother 2013;47:1547-51.

32. Hensey C, Keane D. Clarithromycin induced torsade de pointes. Ir J Med Sci 2008;177:67-8.

33. van Haarst AD, van 't Klooster GA, van Gerven JM, et al. The influence of cisapride and clarithromycin on QT intervals in healthy volunteers. Clin Pharmacol Ther 1998;64:542-6.

34. Hennessy S, Leonard CE, Freeman CP, et al. Validation of diagnostic codes for outpatient-originating sudden cardiac death and ventricular arrhythmia in Medicaid and Medicare claims data. Pharmacoepidemiol Drug Saf 2010;19:555-62.

35. Tamariz L, Harkins T, Nair V. A systematic review of validated methods for identifying ventricular arrhythmias using administrative and claims data. Pharmacoepidemiol Drug Saf 2012; 21(Suppl 1):148-53.

36. De Bruin ML, van Hemel NM, Leufkens HG, et al. Hospital discharge diagnoses of ventricular arrhythmias and cardiac arrest were useful for epidemiologic research. J Clin Epidemiol 2005; 58:1325-9.

37. Quan H, Li B, Saunders LD, et al. Assessing validity of ICD-9-CM and ICD-10 administrative data in recording clinical conditions in a unique dually coded database. Health Serv Res 2008;43:1424-41.

38. Jha P, Deboer D, Sykora K, et al. Characteristics and mortality outcomes of thrombolysis trial participants and nonparticipants: a population-based comparison. J Am Coll Cardiol 1996;27:1335-42.

39. Rosenbaum $P$, Rubin D. The central role of the propensity score in observational studies for causal effects. Biometrika 1983;70:41-55.

40. Austin PC. An introduction to propensity score methods for reducing the effects of confounding in observational studies. Multivariate Behav Res 2011;46:399-424.

41. Austin PC, Grootendorst P, Anderson GM. A comparison of the ability of different propensity score models to balance measured variables between treated and untreated subjects: a Monte Carlo study. Stat Med 2007;26:734-53.

42. Brookhart MA, Schneeweiss S, Rothman KJ, et al. Variable selection for propensity score models. Am J Epidemiol 2006; 163:1149-56.

43. Austin PC. Using the standardized difference to compare the prevalence of a binary variable between two groups in observational research. Commun Stat Simul Comput 2009;38:1228-34.

44. Hirji KF, Fagerland MW. Calculating unreported confidence intervals for paired data. BMC Med Res Methodol 2011;11:66.

45. CPS 2012: Compendium of pharmaceuticals and specialties: the Canadian drug reference for health professionals. $47 \mathrm{th}$ ed. Ottawa: Canadian Pharmacists Association; 2012

46. Tschida SJ, Guay DR, Straka RJ, et al. QTc-interval prolongation associated with slow intravenous erythromycin lactobionate infusions in critically ill patients: a prospective evaluation and review of the literature. Pharmacotherapy 1996;16:663-74.

47. Lee KL, Jim MH, Tang SC, et al. QT prolongation and torsades de pointes associated with clarithromycin. Am J Med 1998;104:395-6.

48. Arellano-Rodrigo E, Garcia A, Mont L, et al. Torsade de pointe and cardiorespiratory arrest induced by azithromycin in a patient with congenital long QT syndrome [article in Spanish]. Med Clin (Barc) 2001;117:118-9.

49. Samarendra P, Kumari S, Evans S, et al. QT prolongation associated with azithromycin/amiodarone combination. Pacing Clin Electrophysiol 2001;24:1572-4.

50. Hayashi Y, Ikeda U, Hashimoto T, et al. Torsades de pointes ventricular tachycardia induced by clarithromycin and disopyramide in the presence of hypokalemia. Pacing Clin Electrophysiol 1999;22:672-4

51. Granowitz EV, Tabor KJ, Kirchhoffer JB. Potentially fatal interaction between azithromycin and disopyramide. Pacing Clin Electrophysiol 2000;23:1433-5.

52. Fleet JL, Dixon SN, Shariff SZ, et al. Detecting chronic kidney disease in population-based administrative databases using an algorithm of hospital encounter and physician claim codes. $B M C$ Nephrol 2013;14:81.

53. Charlson ME, Pompei P, Ales KL, et al. A new method of classifying prognostic comorbidity in longitudinal studies: development and validation. J Chronic Dis 1987;40:373-83.

54. Quan H, Sundararajan V, Halfon P, et al. Coding algorithms for defining comorbidities in ICD-9-CM and ICD-10 administrative data. Med Care 2005;43:1130-9.

55. Downs SH, Black N. The feasibility of creating a checklist fo the assessment of the methodological quality both of randomised and non-randomised studies of health care interventions. J Epidemiol Community Health 1998;52:377-84.

56. Svanström H, Pasternak B, Hviid A. Use of clarithromycin and roxithromycin and risk of cardiac death: cohort study. BMJ 2014;349:g4930.

57. Hancox JC, Hasnain M, Vieweg WV, et al. Azithromycin, cardiovascular risks, QTc interval prolongation, torsade de pointes, and regulatory issues: a narrative review based on the study of case reports. Ther Adv Infect Dis 2013;1:155-65.

58. Gysel M, Vieweg WVR, Hasnain M, et al. Torsades de pointes following clarithromycin treatment. Expert Rev Cardiovasc Ther 2013;11:1485-93.

59. Hancox JC, Hasnain M, Vieweg WV, et al. Erythromycin, QTc interval prolongation, and torsade de pointes: case reports, major risk factors and illness severity. Ther Adv Infect Dis 2014;2:47-59.

60. Albert RK, Schuller JL. Macrolide antibiotics and the risk of cardiac arrhythmias. Am J Respir Crit Care Med 2014;189:1173-80.

61. Hancox JC, Du C, Harchi AE, et al. A novel genetic modifier for clarithromycin-related cardiac arrhythmia risk? Ther Adv Infect Dis 2014;2:71-2.

62. International Epidemiological Association. A dictionary of epidemiology. 5th ed. In: Porta M, editor. New York: Oxford University Press; 2008

63. Azithromycin (systemic) [drug information]. Wolters Kluwer; 2015.

64. Clarithromycin: drug information [drug information]. Wolters Kluwer; 2015.

65. Erythromycin (systemic): [drug information]. Wolters Kluwer; 2015.

Affiliations: Department of Epidemiology and Biostatistics (Trac, Garg), Western University; Lawson Health Research Institute (Trac), London Health Sciences Centre; Institute for Clinical Evaluative Sciences (ICES) Western (McArthur, Jandoc, Dixon, Nash, Garg); Divisions of Clinical Pharmacology (Hackam) and Nephrology (Garg), Department of Medicine, Western University, London, Ont.

Contributors: All of the authors contributed to the study design, development of the data analytic plan and interpretation of the data. Eric McArthur conducted the data analysis Mai Trac, Racquel Jandoc and Amit Garg contributed to the drafting of the manuscript, which all of the authors revised. All of the authors gave final approval of the version to be published and agreed to act as guarantors of the work.

Funding: Academic Medical Organization of Southwestern Ontario.

Data sharing: We cannot share data owing to protection of patients' privacy.

Acknowledgements: Parts of this material are based on data and information compiled and provided by the Canadian Institute for Health Information (CIHI). However, the analyses, conclusions, opinions and statements expressed herein are those of the authors and not necessarily those of CIHI. Amit Garg was supported by the Dr. Adam Linton Chair in Kidney Health Analytics. Research personnel who worked on this project were supported by the Lilibeth Caberto Kidney Clinical Research Unit and the Institute for Clinical Evaluative Sciences Western facility. The authors thank IMS Brogan Inc. for use of their Drug Information Database.

Disclaimer: This study was supported by the Institute for Clinical Evaluative Sciences (ICES), which is funded by an annual grant from the Ontario Ministry of Health and LongTerm Care (MOHLTC). The opinions, results and conclusions reported in this paper are those of the authors and are independent from the funding sources. No endorsement by ICES or the Ontario MOHLTC is intended or should be inferred. 